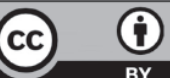

https://creativecommons.org/licenses/by/4.0/

\title{
EL ARTE DE CONSTRUIR MODELOS MATEMÁTICOS DE INVENTARIOS DETERMINÍSTICOS MULTIETAPAS EN MANUFACTURA
}

\section{The art of building mathematical models of multistage deterministic inventories in manufacturing}

CESAR AUGUSTO PINEDA PEREZ ${ }^{1}$, YUDY ALEXANDRA ORJUELA SALAZAR ${ }^{2}$ ANGELA LORENA DIAZ PLAZAS ${ }^{3}$, DAYANA ASTRID ACERO RIVERA ${ }^{4}$

Recibido:14 de mayo de 2020. Aceptado:30 de mayo de 2020

DOI: http://dx.doi.org/10.21017/rimci.2020.v7.n14.a86

\section{Resumen}

Este artículo presenta el procedimiento de elaboración en la construcción de modelos matemáticos de dos etapas en manufactura. Se describe la forma de elaborar los gráficos, sus relaciones y el desarrollo matemático para determinar las variables del modelo.

Palabras clave. Red Logística; Simulación Continua; Inventarios.
\end{abstract}

\section{Aвstract}

This article presents the elaboration procedure in the construction of two-stage mathematical models in manufacturing. The way to elaborate the graphs, their mathematical relationships and the mathematical development to determine the variables of the model are described.

Keywords. Logistics Network; Continuous Simulation; Inventories.

\section{INTRODUCCIÓN}

L factureras es una de las actividades fundamentales que requieren de un manejo y control extremo. Una buena gestión de inventarios redunda en una mejor planeación y programación de la producción, en un eficiente análisis de las capacidades productivas, en una mejor distribución y manejo de los productos terminados, en una mejor coordinación de los inventarios en proceso y de los productos terminados en la red logística, en una reducción de los costos totales de inventario y así sucesivamente podríamos enumerar varios beneficios que se obtienen al aplicar una eficiente y productiva gestión de inventarios en manufactura y logística. La globalización ha obligado a las empresas en adoptar técnicas matemáticas que les permitan reducir costos de manufactura y costos logísticos para poder sobrevivir en un mercado altamente competitivo. Por esta razón, los investigadores y académicos que trabajan en el campo de la investigación de operaciones permanentemente desarrollan modelos matemáticos de inventarios con el objetivo de minimizar costos y lograr una mejor

1 Magister en Ingeniería Industrial, Especialista en Ingeniería de Producción e Ingeniero Industrial de la Universidad Distrital. Docente investigador del grupo O.C.A de la Corporación universitaria republicana. Correo electrónico: cesarpinedaperez@yahoo.es.

2,3,4 Estudiantes de octavo semestre de Ingeniería Industrial de la Corporación Universitaria Republicana y pertenecientes al semillero PMA (programación matemática aplicada) adscrito al grupo de investigación O.C.A. 
coordinación de los inventarios tanto en manufactura (productos en proceso) como en la red logística (producto terminado) [1-7].

Cada empresa manufacturera tiene su propio sistema de inventarios, en particular en relación con los inventarios multietapas manejados en manufactura, en donde se definen y calculan las variables de decisión como los tamaños de lotes óptimos que se manejan entre estaciones o procesos, los niveles óptimos de inventario en cada estación o proceso, los tiempos de producción óptimos, los tiempos de almacenamiento y consumo del inventario óptimos y los periodos de corridas de producción. Asimismo, se determinan los parámetros del modelo matemático de inventario como los costos de alistamiento de la producción, los costos de producción, los costos de mantenimiento del inventario, las tasas de producción y tasas de consumo de cada estación o proceso. Por lo tanto, para construir un modelo matemático multietapas, se requiere caracterizar el sistema multietapa, apoyarse en un gráfico mostrando el comportamiento del inventario y finalmente establecer las relaciones matemáticas del modelo, para luego determinar las expresiones matemáticas de las variables de decisión [8 -14].

\section{Procedimiento para construir MODELOS DE INVENTARIOS MULTIETAPA}

El procedimiento para construir o elaborar modelos de inventarios multietapas es el siguiente:

a) Caracterizar el sistema de inventarios multietapas. Es decir, definir las relaciones de los lotes y tiempos de producción entre los procesos o estaciones de trabajo. Definir si admite o no escasez de inventario. Defina parámetros y variables de decisión.

b) Una vez caracterizado el sistema de inventarios multietapa, proceder a representar este sistema mediante una gráfica que relacione las etapas. Mediante esta gráfico se representa el comportamiento del inventario en los periodos de producción, almacenamiento y agotamiento entre las etapas, procesos o estaciones de producción.

c) Con base en el gráfico, determine las relaciones matemáticas. d) Una vez determinada las relaciones matemáticas, proceda a formular la ecuación de costo total del inventario relacionando todas las etapas.

e) Expresar la ecuación de costo total del inventario (CT) con el mínimo número de variables y derive la función de costo total del inventario con respecto a una variable de decisión e iguálela a cero (derivación parcial para encontrar el tamaño de lote óptimo a un mínimo costo total CT).

f) Obtenga las expresiones matemáticas para cada variable de decisión.

\section{Modelamiento matemático}

\subsection{Caso 1}

Una empresa metalmecánica tipo taller, elabora un producto " $X$ " a través de dos procesos u operaciones en serie: corte y ensamble. Gráficamente el sistema se representa así:

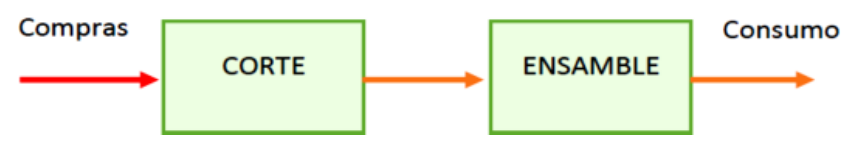

Compras surte toda la materia prima a la estación de corte. Terminada la operación de ensamble, el producto se distribuye de acuerdo con la demanda.

Parámetros:

$T P_{C}=$ tasa de producción en la operación de corte (unidades/unidad de tiempo).

$T P_{E}=$ tasa de producción en la operación de ensamble (unidades/unidad de tiempo).

$\mathrm{T}_{\mathrm{C}}=$ tasa de consumo (unidades/unidad de tiempo).

$C A_{C}=$ Costo de alistamiento de la corrida de producción en la operación de corte (\$/lote).

$C A_{E}=$ Costo de alistamiento de la corrida de producción en la operación de ensamble (\$/lote). 


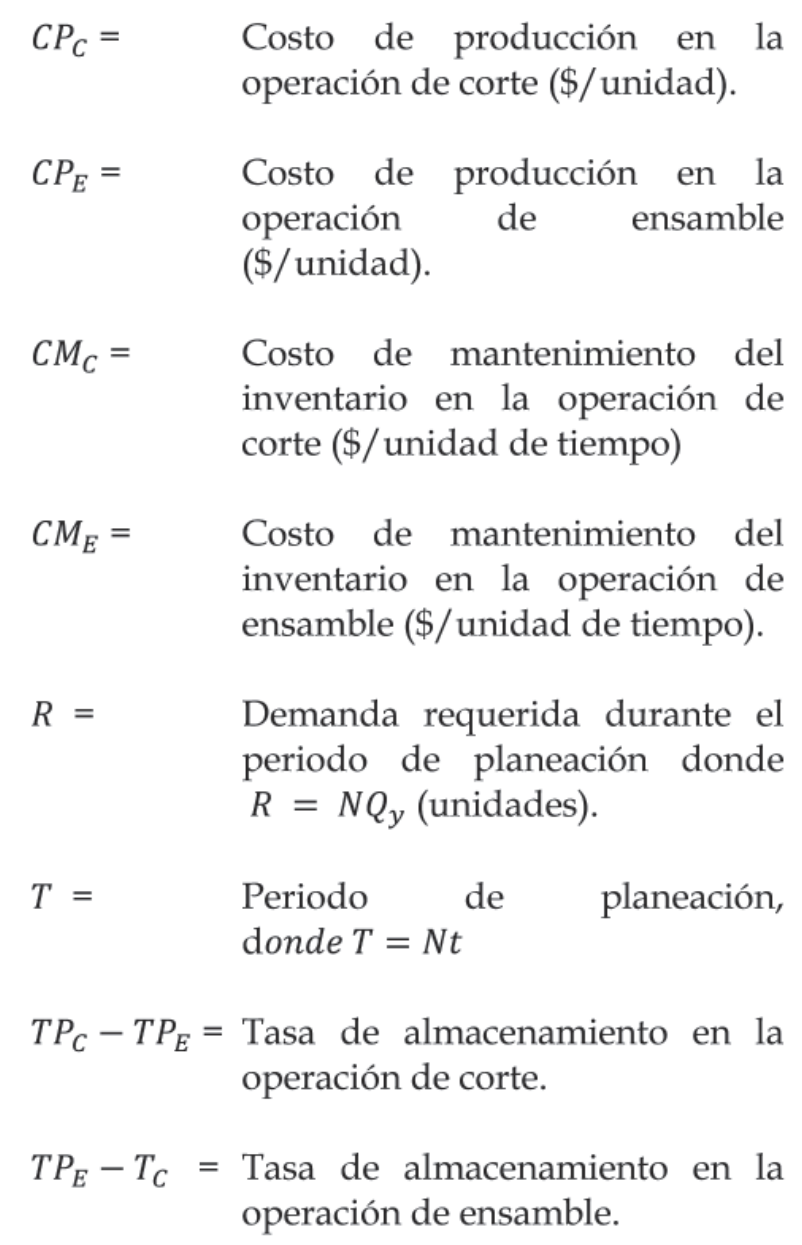

Variables de decisión:

$Q Y_{C}=$ Tamaño del lote de producción en la operación de corte (unidades).

$Q Y_{E}=$ Tamaño del lote de producción en la operación de ensamble (unidades).

$Q_{y}=$ Tamaño del lote de producción optimo común a ambas operaciones (unidades) de acuerdo con los supuestos del modelo.
$Q X_{C}=$ Nivel de inventario optimo en la operación de corte (unidades).

$Q X_{E}=$ Nivel de inventario optimo en la operación de ensamble (unidades).

$t_{1}=$ Periodo de producción de un lote de tamaño " $Q y$ " o tiempo donde se almacena un lote de tamaño " $Q X_{C}$ " en la operación de corte (unidades de tiempo) a una tasa $\left(T P_{C}-T P_{E}\right)$.

$t_{2}=$ Periodo de producción de un lote de tamaño " $Q_{y}$ " o tiempo donde se almacena un lote de tamaño " $Q X_{E}$ " en la operación de ensamble (unidades de tiempo) a una tasa $\left(T P_{E}-T_{C}\right)$.

$t_{2}-t_{1}=$ Período ocioso de producción por ciclo productivo o tiempo de consumo de un lote de tamaño " $Q X_{C}$ " en la operación de corte a una tasa $T P_{E}$.

$t_{3}=$ Período ocioso de producción por ciclo productivo o tiempo de consumo de un lote de tamaño " $Q X_{E}$ " en la operación de ensamble a una tasa $\mathrm{T}_{\mathrm{C}}$.

$t=$ Tiempo entre corridas de producción o duración de un ciclo productivo en donde se consume un lote de tamaño " $Q_{y}$ " del lote de producción (unidades).

$N=$ Numero de lotes que se fabrican durante el periodo planeado.

Supuestos del modelo:

a) $Q Y_{C}=Q Y_{E}=Q_{y}$

b) $T P_{C}>T P_{E}>T_{C}$

La grafica correspondiente a este modelo es:

La gráfica correspondiente a este modelo es la 1.

De acuerdo con el grafico 1, se establecen las relaciones matemáticas:

* En el periodo $t_{1}$ se genera el inventario $Q X_{C}$ a una tasa de almacenamiento $T P_{C}-T P_{E}$, así:

$$
Q X_{C}=\left(T P_{C}-T P_{E}\right) t_{1}
$$




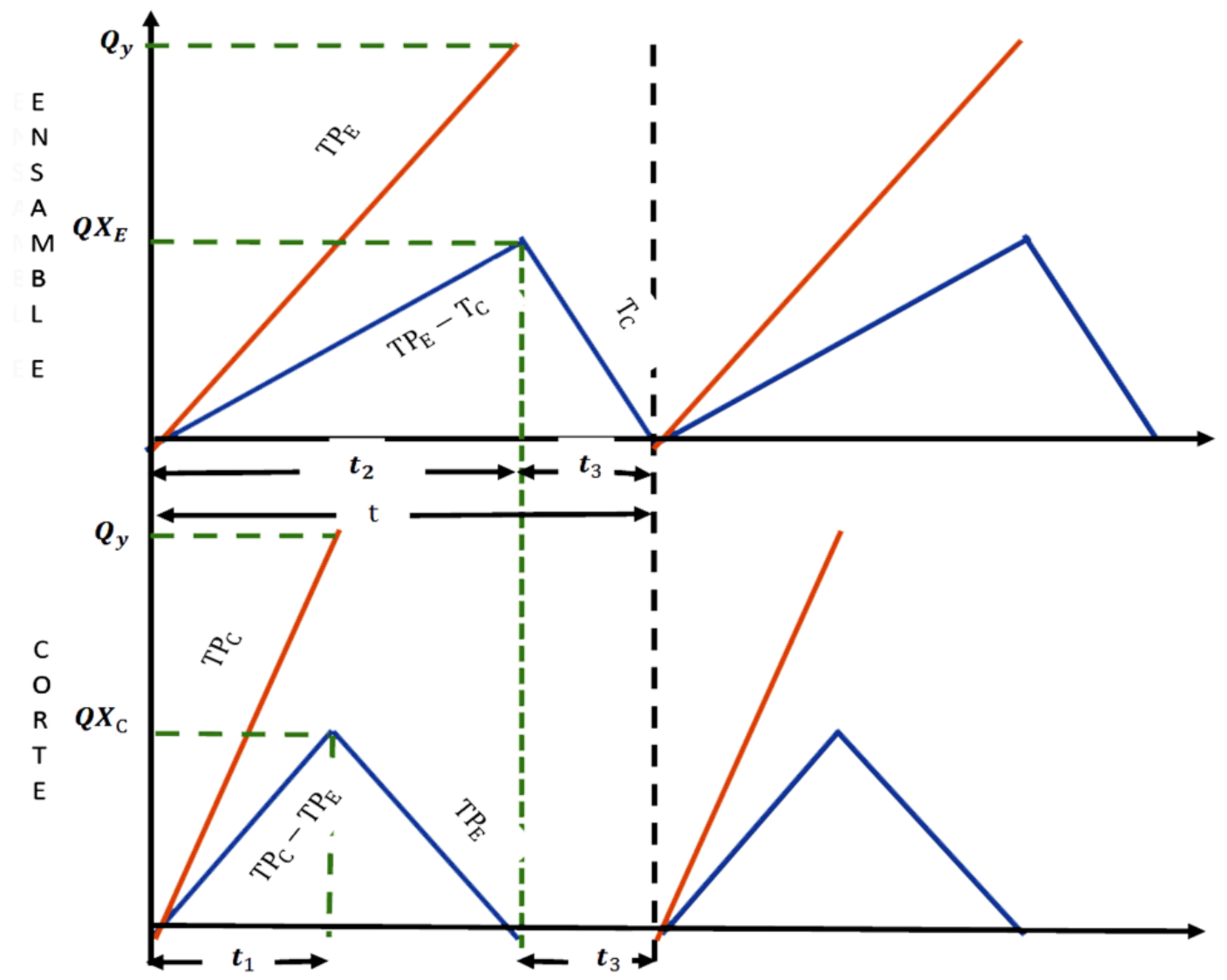

Grafica 1. Representación del caso 1 [Operaciones de corte y ensamble]

* En el periodo $\left(t_{2}-t_{1}\right)$ se consume el inventario $Q X_{C}$ a una tasa $T P_{E}$, así:

$$
Q X_{C}=\left(t_{2}-t_{1}\right) T P_{E}
$$

* En el periodo $t_{2}$ se genera el inventario $Q X_{E}$ a una tasa de almacenamiento $T P_{E}-T_{C}$, asi:

$$
Q X_{E}=\left(T P_{E}-T_{C}\right) t_{2}
$$

* En el periodo $t_{3}$ se consume el inventario $Q X_{E}$ a una tasa $T_{C}$, asi:

$$
Q X_{E}=T_{C} t_{3}
$$

* En el periodo $t_{1}$ se genera el lote de producción $Q_{y}$ en la operación de corte a una tasa de producción $T P_{C}$, así:

$$
Q_{y}=T P_{C} t_{1}
$$

* En el periodo $t_{2}$ se genera el lote de producción $Q_{y}$ en la operación de ensamble a una tasa de producción $T P_{E}$, así:

* Otras relaciones matemáticas:

$$
Q_{y}=T P_{E} t_{2}
$$

$$
t=t_{2}+t_{3}, R=N Q_{y}, N t=T
$$


El siguiente paso es relacionar una variable (en lo posible) en términos de otra variable (haciendo sustituciones) y obtenemos:

$$
\begin{gathered}
t_{1}=\frac{Q_{y}}{T P_{C}}, t_{2}=\frac{Q_{y}}{T P_{E}}, t_{3}=\frac{Q X_{E}}{T_{C}}, t_{2}+t_{3}=\frac{Q_{y}}{T_{C}} \\
Q X_{C}=\frac{\left(T P_{C}-T P_{E}\right) Q_{y}}{T P_{C}}, Q X_{E}=\frac{\left(T P_{E}-T_{C}\right) Q_{y}}{T P_{E}}
\end{gathered}
$$

Ahora, formulamos la ecuación de costos totales (CT) de inventario (Costo total = Costo de producción + costos de alistamiento + costo de mantenimiento del inventario), así:

$$
\begin{gathered}
C T=\left[\left(C P_{C}+C P_{E}\right) Q_{y}+\left(C A_{C}+C A_{E}\right)+\left(\frac{1}{2}\right) C M_{C} Q X_{C} t_{2}+\left(\frac{1}{2}\right) C M_{E} Q X_{E}\left(t_{2}+t_{3}\right)\right] N \\
C T=\left(C P_{C}+C P_{E}\right) Q_{y} N+\left(C A_{C}+C A_{E}\right) N+\left(\frac{1}{2}\right) C M_{C} Q X_{C} t_{2} N+\left(\frac{1}{2}\right) C M_{E} Q X_{E}\left(t_{2}+t_{3}\right) N \\
C T=\left(C P_{C}+C P_{E}\right) Q_{y} \frac{R}{Q_{y}}+\left(C A_{C}+C A_{E}\right) \frac{R}{Q_{y}}+\left(\frac{1}{2}\right) C M_{C} Q X_{C} t_{2} \frac{R}{Q_{y}}+\left(\frac{1}{2}\right) C M_{E} Q X_{E}\left(t_{2}+t_{3}\right) \frac{R}{Q_{y}}
\end{gathered}
$$

Sustituyendo $Q X_{C}, Q X_{E}, t_{2} y t_{3}$ en términos de $Q_{y}$, obtenemos:

$$
\begin{aligned}
C T=\left(C P_{C}+\right. & \left.C P_{E}\right) R+\left(C A_{C}+C A_{E}\right) \frac{R}{Q_{y}}+\left(\frac{1}{2}\right) C M_{C}\left[\frac{Q_{y}\left(T P_{C}-T P_{E}\right)}{T P_{C}}\right]\left(\frac{Q_{y}}{T P_{E}}\right)\left(\frac{R}{Q_{y}}\right) \\
+ & \left(\frac{1}{2}\right) C M_{E}\left[\frac{Q_{y}\left(T P_{E}-T_{C}\right)}{T P_{E}}\right]\left(\frac{Q_{y}}{T_{C}}\right)\left(\frac{R}{Q_{y}}\right)
\end{aligned}
$$

Simplificando la ecuación, obtenemos:

$$
C T=\left(C P_{C}+C P_{E}\right) R+\left(C A_{C}+C A_{E}\right) \frac{R}{Q_{y}}+\left(\frac{1}{2}\right) C M_{C}\left[\frac{Q_{y}\left(T P_{C}-T P_{E}\right)}{T P_{C} T P_{E}}\right] R+\left(\frac{1}{2}\right) C M_{E}\left[\frac{Q_{y}\left(T P_{E}-T_{C}\right)}{T P_{E} T_{C}}\right] R
$$

Reordenando la ecuación:

$$
C T=\left(C P_{C}+C P_{E}\right) R+\frac{\left(C A_{C}+C A_{E}\right) R}{Q_{y}}+\left(\frac{1}{2}\right)\left(\frac{\left(T P_{C}-T P_{E}\right)}{T P_{C} T P_{E}}\right) C M_{C} R Q_{y}+\left(\frac{1}{2}\right)\left(\frac{\left(T P_{E}-T_{C}\right)}{T P_{E} T_{C}}\right) C M_{E} R Q_{y}
$$

Derivamos la ecuación de costo total con respecto a $Q_{y}$ y la igualamos a cero $\left(\frac{\partial C T}{\partial Q_{y}}=0\right)$, obteniendo:

$$
-\frac{\left(C A_{C}+C A_{E}\right) R}{Q_{y}^{2}}+\left(\frac{1}{2}\right)\left(\frac{\left(T P_{C}-T P_{E}\right)}{T P_{C} T P_{E}}\right) C M_{C} R+\left(\frac{1}{2}\right)\left(\frac{\left(T P_{E}-T_{C}\right)}{T P_{E} T_{C}}\right) C M_{E} R=0
$$

Luego, despejando $Q_{y}$, obtenemos:

$$
Q_{y}=\sqrt{\frac{2\left(C A_{C}+C A_{E}\right)}{\left(\frac{\left(T P_{C}-T P_{E}\right)}{T P_{C} T P_{E}}\right) C M_{C}+\left(\frac{\left(T P_{E}-T_{C}\right)}{T P_{E} T_{C}}\right) C M_{E}}}
$$




\subsection{Costos}

Una empresa metalmecánica tipo taller, elabora un producto " $\mathrm{X}$ " a través de dos procesos (proceso 1 y proceso 2) en serie. Compras abastece toda la materia prima al proceso 1 . Terminada la operación del proceso 1, se distribuye el producto de acuerdo con la tasa de consumo.

Supuestos del modelo:
c) $Q Y_{2}=3 Q Y_{1}$
d) $T P_{1}=T P_{2}$
e) $T_{C}<T P_{1}$

Para efectos de manejo hacemos $Q Y_{1}=Q_{y}$ y $Q Y_{2}=3 Q_{y}$

NOTA: Como las tasas de producción del proceso 1 y del proceso 2 son iguales, entonces no se generan inventarios en el proceso 1, por lo tanto, la producción generada en el proceso 1, se envía inmediatamente a surtir el proceso 2 . Entonces, la producción en el proceso 1 no se almacena, porque inmediatamente se consume en el proceso 2, lo que indica que $Q X_{1}=0$.

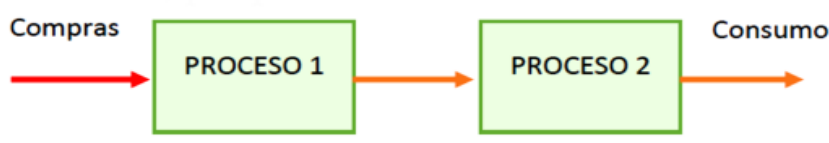

Parámetros:

$T P_{1}=$ tasa de producción en el proceso 1 (unidades/unidad de tiempo).

$T P_{2}=$ tasa de producción en el proceso 2 (unidades/unidad de tiempo).

$\mathrm{T}_{\mathrm{C}}=$ tasa de consumo (unidades/unidad de tiempo).

$C A_{1}=$ Costo de alistamiento de la corrida de producción en el proceso 1 (\$/lote).

$C A_{2}=$ Costo de alistamiento de la corrida de producción en el proceso 2 (\$/lote).

$C P_{1}=$ Costo de producción en el proceso 1 (\$/unidad).

$C P_{2}=$ Costo de producción en el proceso $2 \quad N=$ (\$/unidad).
$C M_{2}=$ Costo de mantenimiento del inventario en el proceso 2 (\$/ unidad de tiempo)

$R=$ Demanda requerida durante el periodo de planeación donde $R=N\left(3 Q_{y}\right)$ (unidades).

$T=$ Periodo de planeación, donde $T=N t$

$T P_{1}-T P_{2}=$ Tasa de despacho del producto " $\mathrm{X}$ " del proceso 1 al proceso 2 (No se almacena en el proceso 1, sino que se envía directamente al proceso 2 ).

$T P_{2}-T_{C}=$ Tasa de almacenamiento en el proceso 2.

Variables de decisión:

$Q Y_{1}=$ Tamaño del lote de producción en el proceso 1 (unidades).

$Q Y_{2}=$ Tamaño del lote de producción en el proceso 2 (unidades).

$Q_{y}=$ Tamaño del lote de producción optimo común a ambos procesos (unidades) de acuerdo con los supuestos del modelo.

$Q X_{2}=$ Nivel de inventario optimo en el proceso 2 (unidades).

$t_{1}=$ Periodo de producción de un lote de tamaño " $Q y$ " en el proceso 1 (unidades de tiempo).

$t_{2}=$ Periodo de producción de un lote de tamaño " $Q y$ " o tiempo donde se almacena un lote de tamaño " $Q X_{2}$ " en el proceso 2 (unidades de tiempo) a una tasa $\left(T P_{1}-T_{C}\right)$.

$t_{3}=$ Período ocioso de producción por ciclo productivo o tiempo de consumo de un lote de tamaño " $Q X_{2}$ " en el proceso 2 a una tasa $\mathrm{T}_{\mathrm{C}}$.

$t=$ Tiempo entre corridas de producción o duración de un ciclo productivo en donde se consume un lote de tamaño " $Q_{y}$ " del lote de producción (unidades).

Numero de lotes que se fabrican durante el periodo planeado.

Rev. Ingeniería, Matemáticas y Ciencias de la Información Vol. 7 / Núm. 14 / julio - diciembre de 2020; pág. 95-103 
La gráfica correspondiente a este modelo es la 2.

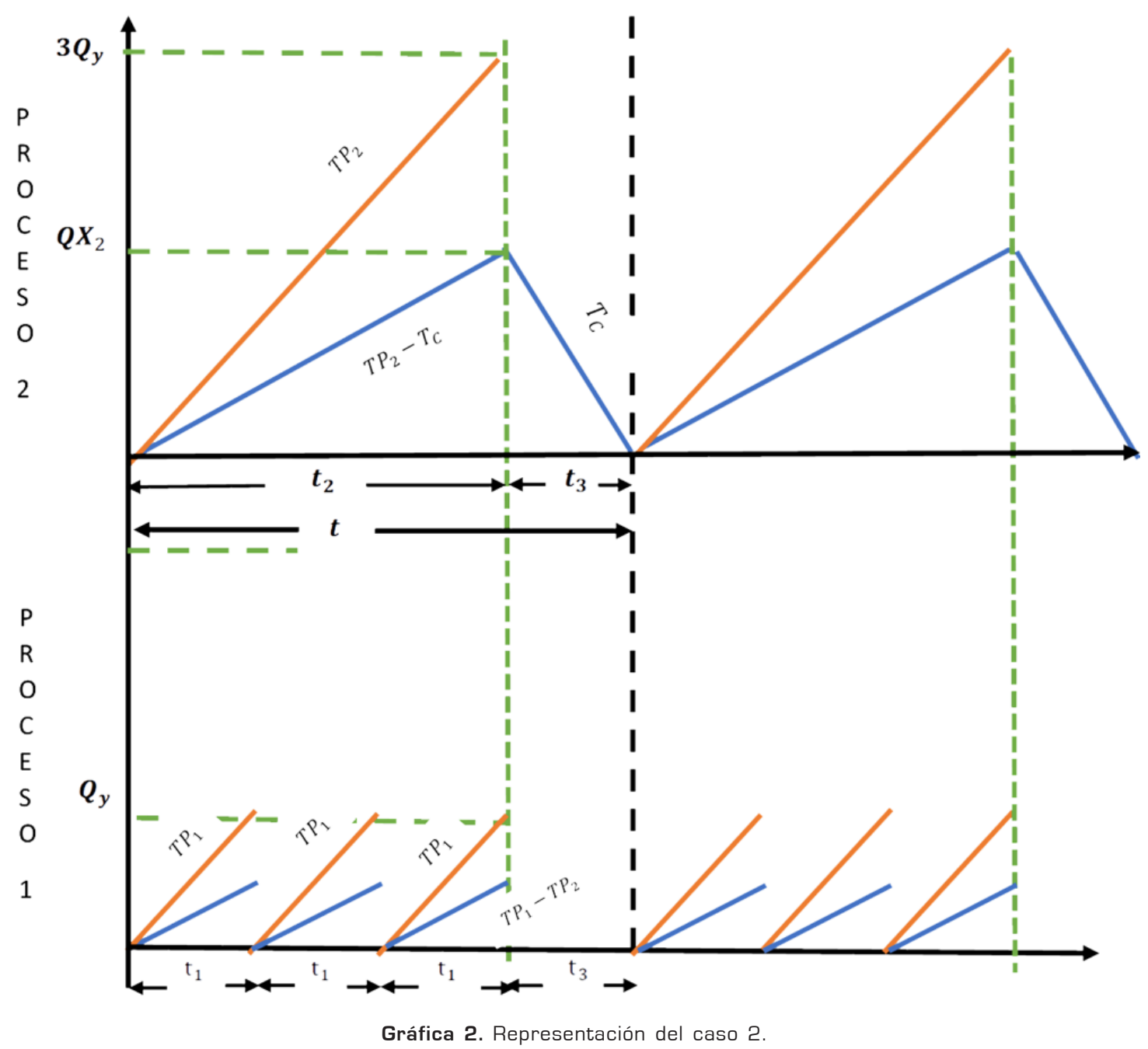

De acuerdo con el grafico 1, se establecen las relaciones matemáticas:

* En el periodo $t_{1}$ se genera el lote de producción $Q_{y}$ en el proceso 1, a una tasa de producción $T P_{1}$, así:

$$
Q_{y}=T P_{1} t_{1}
$$

* En el periodo $t_{2}$ se genera el inventario $Q X_{2}$ a una tasa de almacenamiento $T P_{2}-T_{C}$, asi:

$$
Q X_{2}=\left(T P_{2}-T_{C}\right) t_{2}
$$

* En el periodo $t_{3}$ se consume el inventario $Q X_{2}$ a una tasa $T_{C}$, asi:

$$
Q X_{2}=T_{C} t_{3}
$$

* En el periodo $t_{2}$ se genera el lote de producción $3 Q_{y}$ en el proceso 2, a una tasa de producción $T P_{2}$, así:

$$
3 Q_{y}=T P_{2} t_{2}
$$


Otras relaciones matemáticas:

$$
t=t_{2}+t_{3}, R=N\left(3 Q_{y}\right), N t=T
$$

El siguiente paso es relacionar una variable (en lo posible) en términos de otra variable (haciendo sustituciones) y obtenemos:

$$
\begin{gathered}
t_{1}=\frac{Q_{y}}{T P_{1}}, t_{2}=\frac{3 Q_{y}}{T P_{2}}, t_{2}=\frac{Q X_{2}}{\left(T P_{2}-T_{C}\right)}, t_{3}=\frac{Q X_{2}}{T_{C}}, t_{2}+t_{3}=\frac{3 Q_{y}}{T_{C}} \\
Q X_{2}=\frac{3\left(T P_{2}-T_{C}\right) Q_{y}}{T P_{2}}, N=\frac{R}{3 Q_{y}}
\end{gathered}
$$

Ahora, formulamos la ecuación de costos totales (CT) de inventario (Costo total = Costo de producción + costos de alistamiento + costo de mantenimiento del inventario), así:

$$
\begin{gathered}
C T=\left[\left(C P_{1} Q_{y}+3 C P_{2} Q_{y}\right)+\left(C A_{1}+C A_{2}\right)+\left(\frac{1}{2}\right) C M_{2} Q X_{2}\left(t_{2}+t_{3}\right)\right] N \\
C T=\left(C P_{1} Q_{y}+3 C P_{2} Q_{y}\right) N+\left(C A_{1}+C A_{2}\right) N+\left(\frac{1}{2}\right) C M_{2} Q X_{2}\left(t_{2}+t_{3}\right) N \\
C T=\left(C P_{1} Q_{y}+3 C P_{2} Q_{y}\right) \frac{R}{3 Q_{y}}+\left(C A_{1}+C A_{2}\right) \frac{R}{3 Q_{y}}+\left(\frac{1}{2}\right) C M_{2}\left[\frac{3\left(T P_{2}-T_{C}\right) Q_{y}}{T P_{2}}\right]\left(\frac{3 Q_{y}}{T_{C}}\right)\left(\frac{R}{3 Q_{y}}\right) \\
C T=\frac{C P_{1} Q_{y} R}{3 Q_{y}}+\frac{3 C P_{2} Q_{y} R}{3 Q_{y}}+\left(C A_{1}+C A_{2}\right) \frac{R}{3 Q_{y}}+\left(\frac{1}{2}\right) C M_{2}\left[\frac{3\left(T P_{2}-T_{C}\right) Q_{y}}{T P_{2}}\right]\left(\frac{3 Q_{y}}{T_{C}}\right)\left(\frac{R}{3 Q_{y}}\right) \\
C T=\frac{C P_{1} R}{3}+C P_{2} R+\left(\frac{1}{3}\right) \frac{\left(C A_{1}+C A_{2}\right) R}{Q_{y}}+\left(\frac{3}{2}\right)\left(\frac{C M_{2} R\left(T P_{2}-T_{C}\right) Q_{y}}{T P_{2} T_{C}}\right)
\end{gathered}
$$

Derivamos la ecuación de costo total con respecto a $Q_{y}$ y la igualamos a cero $\left(\frac{\partial C T}{\partial Q_{y}}=0\right)$, obteniendo:

$$
\begin{gathered}
-\frac{\left(C A_{1}+C A_{2}\right) R}{3 Q_{y}^{2}}+\frac{3 C M_{2} R\left(T P_{2}-T_{C}\right)}{2 T P_{2} T_{C}}=0 \\
\frac{\left(C A_{1}+C A_{2}\right) R}{3 Q_{y}^{2}}=\frac{3 C M_{2} R\left(T P_{2}-T_{C}\right)}{2 T P_{2} T_{C}}
\end{gathered}
$$

Luego, despejando $Q_{y}$, obtenemos:

$$
\begin{gathered}
Q_{y}^{2}=\frac{2\left(C A_{1}+C A_{2}\right) T P_{2} T_{C}}{9 C M_{2}\left(T P_{2}-T_{C}\right)} \\
Q_{y}=\sqrt{\frac{2\left(C A_{1}+C A_{2}\right) T P_{2} T_{C}}{9 C M_{2}\left(T P_{2}-T_{C}\right)}}
\end{gathered}
$$




\section{Conclusiones}

La modelización matemática de inventarios multietapas con tasas de consumo o demandas determinísticas, permiten abordar múltiples escenarios con diferentes relaciones entre los tamaños de lotes de producción y las tasas de producción de las diferentes estaciones o procesos de producción en manufactura.

La ventaja de utilizar la matemática de cálculo diferencial multivariable, es que a través de una función de costo total de inventarios, al derivar parcialmente esta función de costo con respecto a alguna o algunas variables, obtenemos no solamente el mínimo costo total de inventarios sino los valores "óptimos" de las variables de decisión (tamaño de lote de producción optimo, niveles de inventarios óptimos, periodos de producción óptimos, periodos de almacenamiento y consumo del inventario óptimos, tiempos de corrida o ciclos de producción óptimos).

Esta técnica procedimental para inventarios multietapas se extiende para " $n$ " etapas o procesos, pero requiere de un análisis sistémico más detallado y una caracterización del sistema de inventarios perfecta y dependiendo de las relaciones matemáticas para más de dos etapas o procesos $(n>2)$, el desarrollo matemático requiere de todos los conceptos teóricos y matemáticos para obtener la mejor coordinación de inventarios en sistemas multietapas.

\section{REFERENCIAS}

[1] D. Zissis, G. Ioannou, and A. Burnetas, "Coordinating Lot Sizing Decisions Under Bilateral Information Asymmetry," Prod. Oper. Manag., doi: 10.1111/poms.13106. 2020.

[2] L. Liu, X. Liu, and D. D. Yao, "Analysis and Optimization of a Multistage Inventory-Queue System," Management Science. doi: 10.1287/ mnsc.1030.0196. 2004.
[3] F. J. C. T. de Ruiter, A. Ben-Tal, R. C. M. Brekelmans, and D. den Hertog, "Robust optimization of uncertain multistage inventory systems with inexact data in decision rules," Comput. Manag. Sci., doi: 10.1007/s10287-016-0253-6. 2017.

[4] D. Gang, C. Li, Y. Z. Li, J. Y. Song, and A. Tanweer, "Optimization on production-inventory problem with multistage and varying demand," J. Appl. Math., doi: 10.1155/2012/648262. 2012.

[5] L. Xin and D. A. Goldberg, "Time (in)consistency of multistage distributionally robust inventory models with moment constraints," Eur. J. Oper. Res., doi: 10.1016/j.ejor.2020.07.041. 2020.

[6] L. Appelgren, H. E. Scarf, D. M. Gilford, and M. W. Shelly, "Multistage Inventory Models and Techniques," Ekon. Tidskr., doi: 10.2307/3438707. 1964.

[7] L. Xin, D. Goldberg, and A. Shapiro, “Distributionally robust multistage inventory models with moment constraints," arXiv Prepr. arXiv1304.3074, 2013.

[8] P. Nagaraj and V.Selladurai, “Analysis of optimum batch size in multistage, multifacility and multiproduct manufacturing systems," Int. J. Adv. Manuf. Technol., doi: 10.1007/s001700200004. 2002.

[9] A. S. Babu and S. G. Deshmukh, "Some studies on integrated multistage production-inventory systems," Eng. Costs Prod. Econ., doi: 10.1016/ 0167-188X(89)90119-5. 1989.

[10] A. Gunasekaran, A. S. Babu, and N. Ramaswamy, "Lot-sizing in a class of multistage production inventory systems," Int. J. Syst. Sci., doi: 10.1080/ 00207729008910549. 1990.

[11] K. Morikawa, K. Takahashi, and D. Hirotani, “Maketo-stock policies for a multistage serial system under a make-to-order production environment," Int. J. Prod. Econ., doi: 10.1016/j.ijpe.2013.02.016. 2014.

[12] M. A. Hoque and S. K. Goyal, "On lot streaming in multistage production systems," Int. J. Prod. Econ., doi: 10.1016/j.ijpe.2003.12.003. 2005.

[13] K. Breithaupt, A. A. Ariel, and D. R. Hare, "Assembling an Inventory of Multistage Adaptive Testing Systems," in Elements of Adaptive Testing, 2009.

[14] D. Rahmani, R. Ramezanian, P. Fattahi, and M. Heydari, "A robust optimization model for multiproduct two-stage capacitated production planning under uncertainty," Appl. Math. Model., doi: 10.1016/j.apm.2013.04.016. 2013. 
